\title{
US panel proposes new name and diagnostic criteria for chronic fatigue syndrome
}

In this News story (BMJ 2015;350:h775, doi:10.1136/bmj.h775), we referred to ME as myalgic encephalitis, when it should have been called myalgic encephalomyelitis.

Cite this as: BMJ 2015;350:h932

๑ BMJ Publishing Group Ltd 2015 